\title{
Ratification vote on taxonomic proposals to the International Committee on Taxonomy of Viruses (2013)
}

\author{
M. J. Adams • A. M. Q. King • E. B. Carstens
}

Received: 19 February 2013/Accepted: 22 February 2013/Published online: 12 April 2013

(C) Springer-Verlag Wien 2013

In accordance with the statutes of the International Committee on Taxonomy of Viruses (ICTV), the final stage in the process of making changes to the Universal Scheme of Virus Classification is the ratification of taxonomic proposals by ICTV Members. This can occur either at a plenary meeting of ICTV, held during an International Congress of Virology conference, or by circulation of proposals by mail followed by a ballot. As in recent years, a list of proposals that had been subjected to the full, multi-stage review process was prepared and presented on the ICTVonline web pages by December 2012. This review process involved input from the ICTV Study Groups and Subcommittees, other interested virologists, and the ICTV Executive Committee. These proposals were submitted to, and discussed by, the ICTV Executive Committee over the past two years and represent changes to virus taxonomy from that published in the Ninth Report [1] and the changes subsequently approved in February 2012 [2]. The ratification process was performed entirely by email. The proposals were sent electronically via email on 14 January 2013 to the 165 members of ICTV, namely the ICTV executive committee members, life members, ICTV subcommittee members and ICTV national representatives. Members were then requested to vote on

M. J. Adams ( $\square)$

Rothamsted Research, Herts, Harpenden AL5 2JQ, UK

e-mail: mike.adams@ rothamsted.ac.uk

A. M. Q. King

Pirbright Laboratory, The Pirbright Institute, Ash Road,

Pirbright, Surrey GU24 0NF, UK

e-mail: amq.king@ pirbright.ac.uk

E. B. Carstens

Department of Biomedical and Molecular Sciences, Queen's

University, Kingston, ON K7L 3N6, Canada

e-mail: carstens@queensu.ca whether or not to ratify the taxonomic proposals (voting closed on 14 February 2013).

The following are the taxonomic proposals that were ratified by ICTV members in February 2013. The proposals are organized by subcommittee, and then the filename posted on-line, which contained the specific information about the proposals. Note that by ICTV convention, all approved taxon names are italicized. The details of all these proposals can be obtained at http://www.ictvonline. org/Official_ICTV_Proposals.htm.

\section{Fungal virus subcommittee}

2011.001a-dF.A.v2.Cafeteriavirus

2011.001aF create species named Cafeteria roenbergensis virus in the new genus Cafeteriavirus, family Mimiviridae

$2011.001 \mathrm{bF}$ create new genus in the family Mimiviridae $2011.001 \mathrm{cF}$ name the genus Cafeteriavirus

$2011.001 \mathrm{dF}$ designate species Cafeteria roenbergensis virus as type species in the new genus

2012.001a-gF.A.v4.Quadriviridae

2012.001aF create species Rosellinia necatrix quadrivirus 1 in the genus Quadrivirus (new), family Quadriviridae (new)

2012.001bF create a new genus in the family Quadriviridae

$2012.001 \mathrm{cF}$ name the new genus Quadrivirus 2012.001dF designate Rosellinia necatrix quadrivirus 1 as the type species of the new genus 
2012.001eF create a new unassigned family

Quadriviridae

2012.001fF name the new family Quadriviridae

2012.001gF assign the new genus Quadrivirus to the new family

2012.004aF.A.v3.Chrysovirus-5sp

2012.004aF create 5 species (Amasya cherry disease associated chrysovirus, Aspergillus fumigatus chrysovirus, Cryphonectria nitschkei chrysovirus 1, Fusarium oxysporum chrysovirus 1 and Verticillium dahliae chrysovirus 1) in the genus Chrysovirus, family Chrysoviridae

\section{Invertebrate virus subcommittee}

2011.001a-gI.A.v2.Mesoniviridae

2011.001aI create species named Alphamesonivirus 1 in the proposed genus Alphamesonivirus, in the proposed family Mesoniviridae in the order Nidovirales

2011.001bI create a new genus in the proposed family Mesoniviridae

2011.001cI name the genus Alphamesonivirus

2011.001dI designate species Alphamesonivirus 1 as type species in the new genus

2011.001eI create a new family in the order Nidovirales

2011.001fI name the family Mesoniviridae

$2011.001 \mathrm{gI}$ assign the proposed genus Alphamesonivirus to the new family

2012.001aI.A.v2.Alphabaculovirus-8sp

2012.001aI create 8 species (Agrotis segetum nucleopolyhedrovirus, Antheraea pernyi nucleopolyhedrovirus, Chrysodeixis chalcites nucleopolyhedrovirus, Clanis bilineata nucleopolyhedrovirus, Euproctis pseudoconspersa nucleopolyhedrovirus, Hyphantria cunea nucleopolyhedrovirus, Leucania separata nucleopolyhedrovirus, and Maruca vitrata nucleopolyhedrovirus) in the genus Alphabaculovirus, family Baculoviridae

\section{Plant virus subcommittee}

2011.005a-eP.A.v2.Zeavirus

2011.005aP create a new genus in the family Tombusviridae

2011.005bP name the genus Zeavirus

$2011.005 \mathrm{cP}$ designate species Maize necrotic streak virus as type species in the new genus

2011.005dP Remove species Maize necrotic streak virus from its current, unassigned, position in the family Tombusviridae

$2011.005 \mathrm{eP} \quad$ Reassign species Maize necrotic streak virus to the new genus

2011.009a-mP.A.v3.split_Necrovirus

2011.009aP create a new genus in the family Tombusviridae

2011.009bP name the genus Alphanecrovirus

2011.009cP designate species Tobacco necrosis virus A as type species in the new genus

$2011.009 \mathrm{dP}$ create a new genus in the family Tombusviridae

$2011.009 \mathrm{eP}$ name the genus Betanecrovirus

2011.009fP designate species Tobacco necrosis virus $D$ as type species in the new genus

2011.009gP Remove species Tobacco necrosis virus A, Olive mild mosaic virus and Olive latent virus 1 from the genus Necrovirus in the family Tombusviridae

2011.009hP Reassign species Tobacco necrosis virus A, Olive mild mosaic virus and Olive latent virus 1 to genus Alphanecrovirus

2011.009iP Remove species Tobacco necrosis virus D, Beet black scorch virus and Leek white stripe virus from the genus Necrovirus in the family Tombusviridae

2011.009jP Reassign species Tobacco necrosis virus D, Beet black scorch virus and Leek white stripe virus to genus Betanecrovirus

2011.009kP Remove species Chenopodium necrosis virus from the genus Necrovirus in the family Tombusviridae

2011.0091P Reassign species Chenopodium necrosis virus to be unassigned in the family Tombusviridae

2011.009mP remove genus Necrovirus from the family Tombusviridae 
2011.010a-eP.A.v3.Gallantivirus

2011.010aP create a new genus in the family Tombusviridae

2011.010bP name the new genus Gallantivirus

2011.010cP designate Galinsoga mosaic virus as type species in the new genus

2011.010dP remove species Galinsoga mosaic virus from the genus Carmovirus in the family Tombusviridae

2011.010eP re-assign species Galinsoga mosaic virus to the new genus Gallantivirus

2011.010f-iP.A.v3.Macanavirus

2011.010fP create species named Furcraea necrotic streak virus in the proposed genus Macanavirus in the family Tombusviridae

2011.010gP create a new genus in the family Tombusviridae

2011.010hP name the new genus Macanavirus

2011.010iP designate Furcraea necrotic streak virus as type species in the new genus

2012.002abP.A.v2.Ampelovirus_rev-sp

2012.002aP create species Grapevine leafroll-associated virus 4 in the genus Ampelovirus, family Closteroviridae

2012.002bP remove species Grapevine leafroll-associated virus 5 from its position in the genus Ampelovirus, family Closteroviridae

2012.003aP.A.v2.Closteroviridae-5spres

2012.003aP Restore 5 species created as unassigned in the family Closteroviridae in 2002 but wrongly omitted from the Master Species List: Olive leaf yellowing-associated virus, Little cherry virus 1, Grapevine leafrollassociated virus 7, Megakepasma mosaic virus and Alligatorweed stunting virus

2012.004aP.A.v3.Tobamovirus-8sp

2012.004aP create 8 species (Bell pepper mottle virus, Cactus mild mottle virus, Clitoria yellow mottle virus, Cucumber mottle virus, Maracuja mosaic virus, Passion fruit mosaic virus, Rattail cactus necrosis- associated virus and Tropical soda apple mosaic virus) in the genus Tobamovirus, family Virgaviridae

2012.005aP.A.v1.Nepovirus-sp

2012.005aP create species Blueberry latent spherical virus in the genus Nepovirus, subfamily Comovirinae, family Secoviridae, order Picornavirales

2012.006aP.A.v2.Fabavirus-sp

2012.006aP create species Cucurbit mild mosaic virus in the genus Fabavirus, subfamily Comovirinae, family Secoviridae, order Picornavirales

2012.007aP.A.v2.Emaravirus-sp

2012.007aP create species Rose rosette virus in the unassigned genus Emaravirus

2012.008aP.A.v1.Nanovirus-sp

2012.008aP create species Faba bean yellow leaf virus in the genus Nanovirus, family Nanoviridae

2012.009aP.A.v1.Crinivirus-sp

2012.009aP create species Diodia vein chlorosis virus in the genus Crinivirus, family Closteroviridae

2012.010aP.A.v2.Benyvirus-sp

2012.010aP create species Rice stripe necrosis virus in the unassigned genus Benyvirus

2012.012aP.A.v1.Tenuivirus-sp

2012.012aP create species Iranian wheat stripe virus in the unassigned genus Tenuivirus

2012.013aP.A.v1.Tritimovirus-sp

2012.013aP create species Yellow oat-grass mosaic virus in the genus Tritimovirus, family Potyviridae 
2012.014aP.A.v3.Endornavirus-sp

$2012.014 \mathrm{aP}$ create species Bell pepper endornavirus in the genus Endornavirus, family

Endornaviridae

2012.015aP.A.v1.Carlavirus_remove-sp

2012.015aP remove species Narcissus symptomless virus from the genus Carlavirus, family

Betaflexiviridae, order Tymovirales

2012.016aP.A.v1.Carlavirus-sp

2012.016aP create species Sweet potato C6 virus in the genus Carlavirus, family Betaflexiviridae, order Tymovirales

2012.017a,bP.A.v2.Alphaflexiviridae-3sp

2012.017aP create species Blackberry virus $E$ to be unassigned in the family Alphaflexiviridae, order Tymovirales

2012.017bP create species Allium virus $X$ and Lagenaria mild mosaic virus in the genus Potexvirus, family Alphaflexiviridae, order Tymovirales

2012.018a-pP.A.v4.Geminiviridae

2012.018aP create species Spinach severe curly top virus in the genus Curtovirus, family Geminiviridae

2012.018bP create species Spinach curly top Arizona virus in the genus Becurtovirus (new), family Geminiviridae

2012.018cP create species Turnip curly top virus in the genus Turncurtovirus (new), family Geminiviridae

2012.018dP create species Eragrostis curvula streak virus in the genus Eragrovirus (new), family Geminiviridae

2012.018eP create a new genus in the family Geminiviridae

2012.018fP name the new genus Becurtovirus

2012.018gP designate Beet curly top Iran virus as the type species of the new genus

2012.018hP create a new genus in the family Geminiviridae

2012.018iP name the new genus Turncurtovirus

2012.018jP designate Turnip curly top virus as the type species of the new genus
2012.018kP create a new genus in the family Geminiviridae

2012.0181P name the new genus Eragrovirus

$2012.018 \mathrm{mP}$ designate Eragrostis curvula streak virus as the type species of the new genus

2012.018nP remove the 4 species Spinach curly top virus, Pepper curly top virus, Beet severe curly top virus and Beet mild curly top virus from their present position in the genus Curtovirus

2012.018oP remove the species Beet curly top Iran virus from its present position in the genus Curtovirus

2012.018pP re-assign the species Beet curly top Iran virus to genus Becurtovirus

2012.019abP.A.v3.Mastrevirus-17sp,rem-2sp

2012.019aP create 17 species (Bromus catharticus striate mosaic virus, Digitaria ciliaris striate mosaic virus, Paspalum dilatatum striate mosaic virus, Oat dwarf virus, Chickpea chlorosis virus, Chickpea chlorosis Australia virus, Chickpea redleaf virus, Chickpea yellows virus, Eragrostis minor streak virus, Digitaria didactyla striate mosaic virus, Chickpea chlorotic dwarf virus, Maize streak Reunion virus, Saccharum streak virus, Paspalum striate mosaic virus, Wheat dwarf India virus, Sporolobus striate mosaic virus 1 and Sporolobus striate mosaic virus 2) in the genus Mastrevirus, family Geminiviridae

2012.019bP remove the species Bean yellow dwarf virus and Setaria streak virus from their present position in the genus Mastrevirus, family Geminiviridae

2012.020a-eP.A.v3.Betaflexiviridae-12sp

2012.020aP create species Banana virus $X$ and Hardenbergia virus $A$ to be unassigned in the family Betaflexiviridae, order Tymovirales

2012.020bP create 6 species (Hippeastrum latent virus, Butterbur mosaic virus, Cucumber veinclearing virus, Helleborus mosaic virus, Hydrangea chlorotic mottle virus, Mirabilis jalapa mottle virus) in the genus Carlavirus, family Betaflexiviridae

2012.020cP create species Asian prunus virus 1 in the genus Foveavirus, family Betaflexiviridae 
2012.020dP create species Phlomis mottle virus in the genus Trichovirus, family Betaflexiviridae

$2012.020 \mathrm{eP}$ create species Actinidia virus $A$ and Actinidia virus $B$ in the genus Vitivirus, family Betaflexiviridae

2012.021aP.A.v1.Trichovirus-sp

$2012.021 \mathrm{aP}$ create species Grapevine Pinot gris virus in the genus Trichovirus, family

Betaflexiviridae

2012.022aP.A.v1.Carlavirus-3sp

2012.022aP create 3 species (Phlox virus S, Phlox virus $B$ and Phlox virus $M$ ) in the genus Carlavirus, family Betaflexiviridae

\section{Prokaryote virus subcommittee}

2011.001a-fB.A.v3.Betafusellovirus

$2011.001 \mathrm{aB}$ create 6 species (Sulfolobus spindle-shaped virus 2, Sulfolobus spindle-shaped virus 4, Sulfolobus spindle-shaped virus 5, Sulfolobus spindle-shaped virus 7 , Sulfolobus spindle-shaped virus 8 , Sulfolobus spindle-shaped virus 9) in the genus Fusellovirus, family Fuselloviridae

$2011.001 \mathrm{bB}$ create 2 species (Sulfolobus spindle-shaped virus 6 and Acidianus spindle-shaped virus 1) in the genus Betafusellovirus (new), family Fuselloviridae

$2011.001 \mathrm{cB}$ create a new genus in the family Fuselloviridae

2011.001dB name the new genus Betafusellovirus

$2011.001 \mathrm{eB}$ designate Sulfolobus spindle-shaped virus 6 as type species in the new genus

2011.001fB change the name of the genus Fusellovirus to Alphafusellovirus

2011.008a-cB.A.v2.Ligamenvirales

$2011.008 \mathrm{aB}$

$2011.008 \mathrm{bB}$

create a new order

$2011.008 \mathrm{cB}$ name the order Ligamenvirales

assign the families Lipothrixviridae and

Rudiviridae to the new order
2011.009a-eB.A.v2.Betaguttavirus

2011.009aB create species named Aeropyrum pernix ovoid virus 1 in the genus Betaguttavirus (new) in the family Guttaviridae

2011.009bB create a new genus in the family Guttaviridae

2011.009cB name the new genus Betaguttavirus

2011.009dB designate Aeropyrum pernix ovoid virus 1 as type species of the genus Betaguttavirus

$2011.009 \mathrm{eB}$ change the name of the genus Guttavirus to Alphaguttavirus

2011.010aB.A.v2.Caudovirales_genus-ren

2011.010aB Rename illegitimate genus names within the order Caudovirales as follows:

2011.010aB "BPP-1-like viruses" to Bppunalikevirus

2011.010aB "Epsilon15-like viruses" to

Epsilon15likevirus

2011.010aB "LUZ24-like viruses" to Luz24likevirus

2011.010aB "N4-like viruses" to N4likevirus

2011.010aB "P22-like viruses" to P22likevirus

2011.010aB "Phieco32-like viruses" to

Phieco32likevirus

$2011.010 \mathrm{aB}$

"PhiKMV-like viruses" to Phikmvlikevirus

$2011.010 \mathrm{aB}$ "SP6-like viruses" to Sp6likevirus

2011.010aB "T7-like viruses" to T7likevirus

2011.010aB "AHJD-like viruses" to Ahjdlikevirus

2011.010aB "Phi29-like viruses" to Phi29likevirus

2011.010aB "I3-like viruses" to I3likevirus

2011.010aB "Mu-like viruses" to Mulikevirus

2011.010aB "P1-like viruses" to Punalikevirus

2011.010aB "PhiH-like viruses" to Phihlikevirus

2011.010aB "PhiKZ-like viruses" to Phikzlikevirus

2011.010aB "T4-like viruses" to T4likevirus

2011.010aB "P2-like viruses" to P2likevirus

$2011.010 \mathrm{aB}$

2011.010aB

2011.010aB

2011.010aB

$2011.010 \mathrm{aB}$

2011.010aB

$2011.010 \mathrm{aB}$

$2011.010 \mathrm{aB}$

$2011.010 \mathrm{aB}$

$2011.010 \mathrm{aB}$

2011.010aB

2011.010aB

2011.010aB

2011.010aB

2011.010aB
"SPO1-like viruses" to Spounalikevirus

Bcep781likevirus to Bcep78likevirus

FelixOllikevirus to Felixounalikevirus

Hapllikevirus to Hapunalikevirus

PBllikevirus to Pbunalikevirus

PhiCD119likevirus to Phicd119likevirus

Hpllikevirus to Hpunalikevirus

"C2-like viruses" to C2likevirus

"L5-like viruses" to L5likevirus

"Lambda-like viruses" to Lambdalikevirus

"N15-like viruses" to N15likevirus

"PhiC31-like viruses" to Phic3unalikevirus

"PsiM1-like viruses" to Psimunalikevirus

"Spbeta-like viruses" to Spbetalikevirus

"T1-likeviruses" to Tunalikevirus 
2011.010aB “T5-like viruses” to T5likevirus

2012.004aB.A.v3.Tunalikevirus_8sp

2012.004aB create 8 species (Escherichia phage Rogue1, Escherichia phage Jk06, Escherichia phage Eb49, Escherichia phage Rtp, Escherichia phage Tls, Cronobacter phage Esp2949-1, Enterobacter phage F20, Shigella phage Shfll) in the genus "T1-like viruses" (proposed name Tunalikevirus), family Siphoviridae, order Caudovirales

2012.005aB.A.v2.T5likevirus-6sp

2012.005bB create 6 species (Escherichia phage T5, Escherichia phage Akfv33, Escherichia phage Eps7, Salmonella phage Spc35, Escherichia phage $B f 23$, Escherichia phage H8) in the genus "T5-like viruses" (proposed name T5likevirus), family Siphoviridae, order Caudovirales

\subsection{6aB.A.v3.PhiKMV-like-2sp}

2012.006aB create species Pantoea phage Limelight and Pantoea phage Limezero in the genus "PhiKMV-like viruses" (proposed name Phikmvlikevirus), subfamily Autographivirinae, family Podoviridae, order Caudovirales

2012.007a-dB.A.v2.Viunalikevirus

2012.007aB create 7 species (Salmonella phage ViI, Salmonella phage Sfp10, Salmonella phage Sh19, Escherichia phage Cba120, Escherichia phage PhaxI, Shigella phage Ag3, Dickeya phage Limestone) in the genus Viunalikevirus (new), family Myoviridae, order Caudovirales

2012.007bB create a new genus in the family Myoviridae

2012.007cB name the new genus Viunalikevirus

$2012.007 \mathrm{~dB}$ designate Salmonella phage ViI as the type species of the new genus

2012.008a-dB.A.v3.Yualikevirus

2012.008aB create 3 species (Pseudomonas phage Yua, Pseudomonas phage M6, Phage phiJl001) in the genus Yualikevirus (new), family Siphoviridae, order Caudovirales
2012.008bB create a new genus in the family Siphoviridae

$2012.008 \mathrm{cB}$ name the new genus Yualikevirus

2012.008dB designate Pseudomonas phage Yua as the type species of the new genus

\section{Vertebrate virus subcommittee}

2010.013aV.A.v3.Ephemerovirus-sp

2010.013aV create species named Kotonkan virus in the genus Ephemerovirus, family Rhabdoviridae, order Mononegavirales

2011.007a-dV.A.v2.Sigmavirus

2011.007aV create 7 species (Drosophila melanogaster sigmavirus, Drosophila obscura sigmavirus, Drosophila affinis sigmavirus, Drosophila tristis sigmavirus, Drosophila immigrans sigmavirus, Drosophila ananassae sigmavirus and Muscina stabulans sigmavirus) in the proposed genus Sigmavirus, family Rhabdoviridae, order Mononegavirales

$2011.007 \mathrm{bV}$ create a new genus in the family Rhabdoviridae, order Mononegavirales

$2011.007 \mathrm{cV}$ name the new genus Sigmavirus

2011.007dV designate Drosophila melanogaster sigmavirus as the type species of the new genus

2011.008a-dV.A.v2.Tibrovirus

2011.008aV create species Tibrogargan virus and Coastal Plains virus in the proposed genus Tibrovirus, family Rhabdoviridae, order Mononegavirales

2011.008bV create a new genus in the family Rhabdoviridae, order Mononegavirales

$2011.008 \mathrm{cV}$ name the new genus Tibrovirus

2011.008dV designate Tibrogargan virus as the type species of the new genus

2011.009aV.A.v3.Ephemerovirus-Sp

$2011.009 \mathrm{aV}$ create species Obodhiang virus in the genus Ephemerovirus, family Rhabdoviridae, order Mononegavirales 
2011.012a-dV.A.v3.Quaranjavirus

2011.012aV create species Quaranfil virus and Johnston Atoll virus in the genus Quaranjavirus (new) in the family Orthomyxoviridae

$2011.012 \mathrm{bV}$ create a new genus in the family Orthomyxoviridae

$2011.012 \mathrm{cV}$ name the new genus Quaranjavirus

2011.012dV designate Quaranfil virus as as the type species of the new genus

2011.015a-dV.A.v1.Aquamavirus

2011.015aV create species Aquamavirus $A$ in the genus Aquamavirus (new) in the family Picornaviridae

$2011.015 b V$ create a new genus in the family Picornaviridae

$2011.015 \mathrm{cV}$ name the new genus Aquamavirus

2011.015dV designate Aquamavirus $A$ as the type species of the new genus

2011.017a-dV.A.v1.Cosavirus

2011.017aV create species Cosavirus $A$ in the genus Cosavirus (new) in the family Picornaviridae

$2011.017 b V$ create a new genus in the family Picornaviridae

$2011.017 \mathrm{cV}$ name the new genus Cosavirus

2011.017dV designate Cosavirus $A$ as the type species of the new genus

2011.018a,bV.A.v2.Enterovirus-Sp,Ren

$2011.018 \mathrm{aV}$ create 2 new species, Enterovirus $F$ and Enterovirus $J$, in the genus Enterovirus, family Picornaviridae

2011.018bV Change the names of the following ten species: Human enterovirus $A$ to Enterovirus A, Human enterovirus $B$ to Enterovirus B, Human enterovirus $C$ to Enterovirus $C$, Human enterovirus $D$ to Enterovirus D, Bovine enterovirus to Enterovirus E, Porcine enterovirus $B$ to Enterovirus $G$, Simian enterovirus $A$ to Enterovirus H, Human rhinovirus $A$ to Rhinovirus A, Human rhinovirus $B$ to Rhinovirus $B$ and Human rhinovirus $C$ to Rhinovirus $C$
2011.019a-dV.A.v2.Megrivirus

2011.019aV create species Melegrivirus $A$ in the genus Megrivirus (new) in the family Picornaviridae

2011.019bV create a new genus in the family Picornaviridae

$2011.019 \mathrm{cV}$ name the new genus Megrivirus

2011.019dV designate Melegrivirus $A$ as as the type species of the new genus

2011.020aV.A.v2.Kobuvirus-Sp

2011.020aV create species Aichivirus $C$ in the genus Kobuvirus, family Picornaviridae, order Picornavirales

2011.021a-dV.A.v1.Salivirus

2011.021aV create species Salivirus $A$ in the genus Salivirus (new) in the family Picornaviridae, order Picornavirales

$2011.021 \mathrm{bV}$ create a new genus in the family Picornaviridae

2011.021cV name the new genus Salivirus

2011.021dV designate Salivirus $A$ as as the type species of the new genus

2011.022a-dV.A.v1.Aurivirus

2011.022aV create species Haliotid herpesvirus 1 in the genus Aurivirus (new) in the family Malacoherpesviridae, order Herpesvirales

$2011.022 \mathrm{bV}$ create a new genus in the family Malacoherpesviridae

$2011.022 \mathrm{cV}$ name the new genus Aurivirus

$2011.022 \mathrm{dV}$ designate Haliotid herpesvirus 1 as as the type species of the new genus

2012.001a-dV.A.v2.Perhabdovirus

2012.001aV create 3 species (Perch rhabdovirus, Anguillid rhabdovirus, Sea trout rhabdovirus) in the genus Perhabdovirus (new), family Rhabdoviridae, order Mononegavirales

$2012.001 b V$ create a new genus in the family Rhabdoviridae

2012.001cV name the new genus Perhabdovirus

2012.001dV designate Perch rhabdovirus as the type species of the new genus 
2012.004aV.A.v3.Arenavirus-sp

2012.004aV create species named Luna virus in the genus Arenavirus, family Arenaviridae

2012.006aV.A.v1.Cytomegalovirus-4sp

2012.006aV create 4 species (Papiine herpesvirus 3, Aotine herpesvirus 1, Saimiriine herpesvirus 4, Cebine herpesvirus 1) in the genus Cytomegalovirus, subfamily Betaherpesvirinae, family Herpesviridae, order Herpesvirales

2012.007aV.A.v1.Alphavirus-sp

2012.007aV create species Madariaga virus in the genus Alphavirus, family Togaviridae

2012.010aV.A.v1.Hantavirus-sp

2012.010aV create species Sangassou virus in the genus Hantavirus family Bunyaviridae

2012.011a-dV.A.v2.Pegivirus

2012.011aV create species Pegivirus $A$ and Pegivirus $B$ in the genus Pegivirus (new), family Flaviviridae

$2012.011 \mathrm{bV}$ create a new genus in the family Flaviviridae

2012.011cV name the new genus Pegivirus

2012.011dV designate Pegivirus $A$ as the type species of the new genus

\subsection{3a-dV.A.v2.Dicipivirus}

2012.013aV create species Cadicivirus $A$ in the genus Dicipivirus (new), family Picornaviridae

$2012.013 \mathrm{bV}$ create a new genus in the family Picornaviridae

2012.013cV name the new genus Dicipivirus designate Cadicivirus $A$ as the type species of the new genus

2012.014aV.A.v1.Kobuvirus-Sp,Ren

2012.014aV rename species Aichi virus and Bovine kobuvirus (family Picornaviridae, genus Kobuvirus) as Aichivirus $A$ and Aichivirus $B$, respectively

2012.016aV.A.v2.Siadenovirus-Sp

2012.016aV create species Skua adenovirus $A$ in the genus Siadenovirus, family Adenoviridae

\section{Conclusion}

All of the above taxonomic proposals were unanimously (or nearly unanimously) approved by the members who voted. The return rate of votes was approximately $41 \%$. There were a small number of objections to the new genus names in the order Caudovirales on the grounds that many of these names are difficult to pronounce. The ICTV Executive Committee understands these objections, but needs also to respect the views of the specialists in this area of virology. These taxonomic changes are now part of the official ICTV taxonomy. An up-to-date list of all approved taxa can be found on the ICTV online web site: http://ictvonline.org under the Taxonomy link.

In addition to these taxonomic changes, a number of changes to the Statutes of ICTV and the International Code of Virus Classification and Nomenclature were also approved. These will be presented in a separate article.

\section{References}

1. King AMQ, Adams MJ, Carstens EB, Lefkowitz EJ (2011) Virus taxonomy. Ninth Report of the International Committee on Taxonomy of Viruses. Elsevier Academic Press, London

2. Adams MJ, Carstens EB (2012) Ratification vote on taxonomic proposals to the International Committee on Taxonomy of Viruses (2012). Arch Virol 157:1411-1422 